\title{
Grape Seed Proanthocyanindin Extract Moderated Retinal Pigment Epithelium Cellular Senescence Through NAMPT/SIRT1/NLRP3 Pathway
}

\section{Yan Wu}

Zhengzhou University First Affiliated Hospital

\section{Sanyou Dai}

Lixiang Eye Hospital of Soochow University

\section{Yang Long}

Zhengzhou University First Affiliated Hospital

Hongzhuo Liu

Zhengzhou University First Affiliated Hospital

\section{Weiwei Wan}

Zhengzhou University First Affiliated Hospital

\section{Guangming Wan}

Zhengzhou University First Affiliated Hospital

\section{Wencui Wan}

Zhengzhou University First Affiliated Hospital

Wei Zhu ( $\nabla$ shzhuwei0722@163.com )

Changshu Hospital Affiliated to Soochow University: First People's Hospital of Changshu City https://orcid.org/0000-0001-7721-7193

\section{Research}

Keywords: cellular senescence, retinal pigment epithelium, nicotinamide phosphoribosyltransferase, Sirtuin 1, inflammasome, NLRP3

Posted Date: February 25th, 2021

DOl: https://doi.org/10.21203/rs.3.rs-230519/v1

License: (c) (1) This work is licensed under a Creative Commons Attribution 4.0 International License. Read Full License 


\section{Abstract}

Background: Cellular senescence of retinal pigment epithelium (RPE) cell was an important cause of degenerative retinal disorders, however, the potential effects of grape seed proanthocyanindin extract (GSPE) through regulating NAMPT/SIRT1/NLRP3 pathway remained unclear.

Methods: The effects of GSPE on the cellular senescence biomarkers as well as NAMPT and NAD+ contents were detected in both in-vivo and in-vitro RPE cell models. The protection of GSPE treatment on the mitochondrial homeostasis and barrier function of RPE cells were detected with mtDNA lesions, JC-1 staining, ZO1 expression, trans-epithelial cell resistance (TEER) as well as senescence-associated secretory phenotype (SASP) expressions. The GSPE treatment with NAMPT inhibitor, Fk866, and SIRT1 inhibitor, EX-527, was used in the potential NAMPT/SIRT1/NLRP3 mechanism detection.

Results: GSPE significantly improve the NAMPT and NAD+ content in aging mice and thus alleviated the RPE cellular senescence. In advanced in-vitro studies, GSPE could be an activator of NAMPT and thus relieved $\mathrm{H}_{2} \mathrm{O}_{2}$ induced $\mathrm{NAD}+$ depression. In advanced analyses, it was reported that GSPE could alleviate mitochondrial homeostasis, barrier function and SASP of aging RPE cells. Thus, detection the SASP in invitro aging model provided us knowledge in the understanding of the anti-aging role of GSPE and following detailed pathological mechanism analyses demonstrated that GSPE demonstrated the protective effects in aging RPE cells through NAMPT/SIRT1/NLRP3 pathway.

Conclusions: These findings indicate that GSPE alleviated cellular senescence both in-vivo and in-vitro through NAMPT/SIRT1/NLRP3 pathway. This study highlighted the importance both the potential GSPE in degenerative retinopathy as well as the crosstalk of NAD+ metabolism, SIRT1 function and NLRP3 activation.

\section{Introduction}

Cellular senescence, which was an important cause of physiological and pathological changes in the ocular structure including the retina [1], was responsible for the development of diabetic retinopathy (DR), retinitis pigmentosa (RP) and age-related macular degeneration (AMD). The cellular senescence related retinal degenerative disorders would affect the visual function of more than 500 million patients, even lead to blindness in severe cases [2]. RPE was a polygonal cell layer located between the choroid and the neuroretina and its dysfunction was regarded as the key factor leading to the occurrence of AMD, DR and $\operatorname{RP}[3,4]$. The senescence of RPE cells would impair its phagocytosis and barrier function, along with abnormal secretion of neurotrophic and angiogenic factors [5]. Therefore, clarifying the mechanism of RPE cell senescence will help to deepen the understanding of the pathogenesis of retinal degenerative diseases, and thus provide clues for the development of new drugs for related diseases, which was a major problem that needs to be solved urgently.

Nicotinamide adenine dinucleotide (NAD+), which was the core cofactor of mitochondrial function, played a vital role in regulating cell metabolism and energy homeostasis and was reported to be a key 
target for breakthroughs in current anti-aging field [6, 7]. The decline of NAD + content with age was closely related to the occurrence of age-related degenerative diseases [6, 8]. In blue-light induced retinal damage, DR and genetic glaucoma animal models, it was found that NAD + contents in the retina were decreased, and exogenous supplementation of NAD + could significantly prevent age-related NAD + decrease and retinal dysfunction $[9,10]$. A recent study demonstrated that NAD + in RPE cells of AMD patients was decreased significantly by analyzing the metabolic pathways in the donated eyes of AMD patients. Nicotinamide phosphoribosyltransferase (NAMPT), also known as visfatin and pre-B cell enhancing factor (PBEF), was the rate-limiting enzyme that catalyzes the synthesis of nicotinamide into nicotinamide mononucleotide (NMN), which was the direct precursor of NAD + in the NAD salvage synthesis pathway [11]. In retinal degenerative diseases, the expression of NAMPT and the NAD + content was consistent. Therefore, we conjectured that abnormal NAD + metabolism was involved in RPE cellular senescence, and thus the key regulatory factors of NAD + metabolic pathway, NAMPT, was related with the development of retinal degenerative disorders.

Grape seed proanthocyanindin extract (GSPE) was extracted from whole grape seeds and regarded as an important source of polyphenolic bioflavonoid [12]. As reported in the previous studies, GSPE exhibited a variety of properties, such as antioxidant, anti-inflammatory and as well as anti-aging $[13,14]$. GSPE could up-regulate the concentration of NAMPT [15], however, the potential protective role of GSPE induced NAMPT on the RPE cellular senescence has not been studied. Therefore, based on the foregoing viewpoints, we hypothesized that GSPE treatment could inhibit the RPE cellular senescence through upregulation of NAMPT. Advanced in-vivo and in-vitro studies would be conducted to detect the effects of GSPE on the RPE cell function as well as the potential pathological mechanisms through NAMPT/SIRT1/NLRP3 pathway.

\section{Materials And Methods}

Regents

The GSPE with over $98 \%$ proanthocyanindin was purchased from Rongsheng Biotechnology Co., Ltd. (Xi'an, China) and dissolved in drinking pure water for in-vivo study and in DMSO for in-vitro treatment. NAMPT inhibitor, Fk866, and SIRT1 inhibitor, EX-527, were purchased from Sigma (Sigma-Aldrich, USA). The NAD + precursor, NMN, was purchased from APExBIO (APExBIO, USA).

Animals

All animal experiments were conducted in accordance with the Association for Research in Vision and Ophthalmology Statement for use of Animals in Ophthalmic and Vision Research. The animal protocol was approved by the Animal Ethics Committee of Zhengzhou University. Both male and female C57BL/6J mice of different age groups were obtained from a commercial vendor (Sipuer-Bike Laboratory, Shanghai). The mice would be housed under a pathogen-free environment with a 12:12 h light/dark cycle and free access to laboratory chow and water. Animal rooms were maintained at $20-22^{\circ} \mathrm{C}$ with $30-70 \%$ relative humidity. Blood samples collections and RPE isolations were conducted in accordance with the 
previous studies in different time points. The obtained animal samples were stored in $-80{ }^{\circ} \mathrm{C}$ until advanced experiments. GSPE treatment would be conducted through added GSPE in drinking water and GSPE treatment conducted in the 15 months aged mice. After three months GSPE treatment, the 18 months aged mice would be used in the following experiments.

Cell culture

The ARPE19 cells were purchased from American type culture collection (ATCC) and cultured with Dulbecco's modified Eagle medium (DMEM) (HyClone, USA) supplemented with 10\% heated-inactivated fetal bovine serum (FBS), $100 \mathrm{U} / \mathrm{ml}$ penicillin, and $100 \mu \mathrm{g} / \mathrm{ml}$ streptomycin (Gibco, USA). The ARPE-19 cells would be maintained at $37^{\circ} \mathrm{C}$ in a humidified chamber with $5 \% \mathrm{CO}_{2}$ and $1: 3$ subcultures were conducted using trypsin-EDTA solution.

RNA extraction and real-time PCR

The extraction of total RNA of both animal examples and RPE cells were conducted with a Trizol reagent (Invitrogen, USA) following the manufacturer's protocol. cDNA synthesis was conducted using Transcriptor First Strand cDNA Synthesis Kit (Thermo Fisher Scientific, USA). RT-PCR was performed with Applied Biosystem ${ }^{T M}$ SYBR ${ }^{T M}$ using the TaqMan Multiplex Real-Time PCR Solution according to the manufacturer's instructions. Relative mRNA expressions would be calculated using the ${ }^{\Delta \Delta} \mathrm{Ct}$ method. The primers used in this study were listed in Table 1. Three independent experiments were conducted for each sample and data were used for advanced analyses.

NAD + assay

The NAD + contents in both animal samples and cellular homogenates were detected using a commercial NAD + assay kit (Sigma-Aldrich, USA). All the experiments were conducted according to the manufacturer's instructions.

In-vitro aging model

The in-vitro aging model of RPE cells were conducted according to a protocol as previously described in our lab [16] with slight modifications. In general, the APRE-19 cells were placed in petri dishes and then exposed to $800 \mu \mathrm{M} \mathrm{H}_{2} \mathrm{O}_{2}$ for 2 hours at a temperature of $37^{\circ} \mathrm{C}$ from day one for five continuous days. Fresh complete media would be replaced after $\mathrm{H}_{2} \mathrm{O}_{2}$ treatment. The aging RPE cells would be collected in the other day after last $\mathrm{H}_{2} \mathrm{O}_{2}$ treatment and this aging in-vitro model would be used in the following studies.

Cell viability

The cell viability was examined in this study with MTT assay. MTT dye (Solarbio, China) was added to the 96-well plate with ARPE-19 cells in different groups and incubated for four hours at $37^{\circ} \mathrm{C}$. After replacing the culture media with $150 \mu \mathrm{L}$ of DMSO to each well, the 96 -cell plates were shocked thoroughly 
until the crystals dissolved. Absorbance was measured at a wavelength of $490 \mathrm{~nm}$ using the Thermo Scientific Fluoroskan microplate reader (Thermo Fisher Scientific, USA). Three independent experiments were conducted for each group and data were used for advanced analyses.

$\beta$-galactosidase assay

Cellular senescence marker, beta-galactosidase ( $\beta-\mathrm{Gal})$, was used in the measurement of senescent status in different cell models and the assay was performed with a commercia assay kit (Beyotime Biotechnology, China) according to the manufacturer's protocols. The view and counting would be conducted under the brightfield in Leica Microscope (Leica Biosystems, Germany). The senescent cells expressing SA $\beta$-gal were positive cells and stained blue. The positive cells rates were recorded in each group and the data would be used in advanced analyses.

mtDNA lesions measurement

As NAD + was an important contributor in mtDNA damage repairment, the mtDNA lesion measurement was conducted in each group. The detection of mtDNA was performed using a well-validated QPCR based detection assay, as previously described [17]. The DNA extraction was conducted using a genomictip kit (Qiagen, CA) and the extracted DNA would be stored in $-80{ }^{\circ} \mathrm{C}$ before experiments. The principle of mtDNA lesion measurement was the that any lesion on a DNA template would block the function of thermostable polymerase and therefore only the DNA templates without any DNA damages could be amplified. After DNA templates quality control, the QPCR would be conducted with a High-Stability PCR Kit (Genscript, China). The $\beta$-globin product was used as an inner-control for the detection of relative mtDNA lesion as described previously. Three independent experiments were conducted with three repetitions.

Mitochondrial membrane potential

The JC-1 dye accumulated in the mitochondria in a potential-dependent manner and could be used to detect the membrane potential of cells, tissues or purified mitochondria. JC-1 was an ideal fluorescent probe widely used to detect the $\triangle \Psi \mathrm{m}$ position of mitochondrial membrane electricity with a JC-1 assay kit (Beyotime Biotechnology, China). After aspirate culture media in the 6-well plate and washing the cells with PBS, $1 \mathrm{ml} \mathrm{JC}-1$ dyeing working solution was added each well. After incubating the mixed working system for 20 minutes at $37^{\circ} \mathrm{C}$, the cells would be washed with the JC-1 Buffer $(1 \mathrm{x})$ and placed on ice. The cells would be observed under a confocal microscope (Leica, Germany). The polymer emits strong red fluorescence $(E x=585 \mathrm{~nm}, E m=590 \mathrm{~nm})$ and the monomers produced green fluorescence $(E x=514$ $\mathrm{nm}, \mathrm{Em}=529 \mathrm{~nm}$ ). The fluorescence rate of monomers/polymer was detected and analyzed.

Trans-epithelial cell resistance measurement

Trans-epithelial cell resistance (TEER) was used to identify the cellular permeability and it was conducted with TEER24 (Applied Biophysics, USA). ARPE-19 cells were inoculated on the bottom membrane of the 
upper layer of the transwell chamber $\left(0.3 \times 10^{4} /\right.$ well), and $1.2 \mathrm{ml}$ of complete cell culture medium was added to the chamber. The TEER was measured after the cell was confluent. After debugging the TEER24 epithelial volt ohmmeter and wiping the electrode pads with alcohol, the electrode sheets were immersed into the inner and outer culture medium, respectively. The resistance of the blank control group (without any cells inoculated) and the experimental group was measured vertically in sequence. The measurement was repeated three times for each hole, and three multiple holes were set for each group. Calculation of TEER value was conducted as following: TEER=(resistance value experimental group -resistance $_{\text {blank control }}$ ) $\times 0.3$ and the unit is $\Omega \mathrm{cm}^{2}$.

\section{Cellular immunofluorescence}

After plating the ARPE-19 cells into six-well plates and generating cells grown on coverslips, the cover slides were washed once with PBS buffer and $1 \mathrm{~mL}$ of immunostaining fixative was fixed for $20 \mathrm{~min}$ at room temperature. To break the membrane, $0.2 \%$ Triton X-100 was added after three times washes with PBS. Incubation with blocking solution (PBS containing $10 \%$ goat serum) was conducted for $2 \mathrm{~h}$ at $4^{\circ} \mathrm{C}$ and then the cells would be incubated with dilute primary antibody (anti-ZO1 and anti-NLRP3) for immunostaining in a cold room overnight. The cells were washed three times with a PBS and then the secondary antibody was added incubate with the cells for $1 \mathrm{~h}$ in the dark. Drop the DAPI \& anti-quencher mixture onto the glass slide and flip the cover glass. Observation and record of the immunofluorescence photographs were conducted with a Zeiss confocal microscope (Leica Biosystems, Germany) under dark.

Western blot

After the total protein extracted from tissues and cellular samples with RIPA buffer with protease inhibitors, BCA quantitative measurement of protein concentration was conducted. The relative expression of the target protein was normalized to $\beta$-actin. A total of $20 \mu \mathrm{g}$ protein for each sample was separated by SDS-PAGE and transferred onto PVDF membrane (Millipore, USA). After incubating with the milk in TBS for blocking, the membrane would be incubated the corresponding primary antibody, including anti-p16 ${ }^{\text {INK4a }}$, p21 Waf/Cip1, SIRT1, NLRP3, apoptosis-associated speck-like protein (ASC), procaspase- 1 , caspase 1 and $\beta$-actin, at $4^{\circ} \mathrm{C}$ overnight. On the second day, the membrane was washed three times with TBST and incubated with the secondary antibody for $1 \mathrm{~h}$. The density of the bands was quantified using Labworks image acquisition software (UVP, USA) and were quantitated using ImageJ software ( $\mathrm{NIH}, \mathrm{USA})$.

ELISA assays

IL-1 $\beta$ and IL18 production in the supernatant in ARPE-19 cells of different groups were measured using IL$1 \beta$ and IL-18 ELISA kit (BioPioneer Tech, China) according to the manufacturer's instructions. Samples were quantified with a corrected value of 450 and $570 \mathrm{~nm}$, reading the absorbance measurements in the Thermo Scientific Fluoroskan microplate reader (Thermo Fisher Scientific, USA). Three replications for all the samples were adopted and the data of three independent studies were used in final analyses. 


\section{Statistical analysis}

The data was expressed as a mean \pm stand difference (SD) in this study. The difference between groups was analyzed using non-paired t test and the difference between over two groups were analyzed using one-way analysis of variance (ANOVA) followed by the newman-keuls method for multiple comparison analysis test. The $P$ value $<0.05$ denotes statistical significance.

\section{Results}

Decreased NAMPT and NAD + levels in RPE and plasma could be improved by GSPE treatment in aging mouse

$N A D+$ metabolism was a key regulator in the increasing of aging and salvage pathway provided about $85 \%$ of total NAD+. As described in Fig. 1A, NAD + was transformed into NAM through NAD + consume. Then, the NAM would become NMN after being catalyzed by NAMPT. NMN would also be transformed into NAD + by NMNAT1-3 enzyme and the cycle of NAD + salvage was completed. Comparing with the young mice (2 months aged), lower NAMPT expression in 18-month aged mice and decreased NAD + contents in 12- and 18-months aged mice were detected $(P<0.05)$. After supplementing with GSPE at a concentration of $250 \mathrm{mg} / \mathrm{kg}$ from 15 to 18 months age, significant increased NAMPT expression and NAD + content was detected $(P<0.05$, Fig. 1B and 1C). A similar expression pattern was observed in the mouse plasma and NAMPT/NAD + levels decline was associated with increasing age in mouse plasma. The intake of $250 \mathrm{mg} / \mathrm{kg}$ GSPE for three months significantly improved the NAMTP and NAD + content in plasma $(P<0.05$, Fig. 1D and 1E).

GSPE alleviated aging related cellular senescence and SASP in mouse RPE

To detect the effect of GSPE supplement on the aging of mice, the expressions of cellular senescence related markers, $\mathrm{p} 16^{\mathrm{INK} 4 \mathrm{a}}$ and $\mathrm{p} 21^{\mathrm{Waf} / \mathrm{Cip} 1}$, as well as the SASP status was reported. As showed in Fig. 2A, $\mathrm{p} 16^{\mathrm{INK} 4 \mathrm{a}}$ and $\mathrm{p} 21^{\text {Waf/Cip } 1}$ expressions were increased in the aging mice (18 months aged) and the GSPE in $250 \mathrm{mg} / \mathrm{kg}$ would decline these cellular senescence biomarkers. As SASP involved a serious of proinflammatory factors, chemokines as well as proteases, the expression of tumor necrosis factor-a (TNFa), transforming growth factor- $\beta 1$ (TGF- $\beta 1$ ), interleukin-1 $\beta$ (IL1 $\beta$ ), interleukin-6 (IL6), interleukin-8 (IL8), interleukin-18 (IL18), monocyte chemotactic protein 1 (MCP-1), granulocyte-colony stimulating factor (GCSF), chemokine (C-X-C motif) ligand 1 protein (CXCL1), chemokine (C-X-C motif) ligand 2 protein (CXCL2), inositol requiring enzyme1a (Ire1a), plasminogen activator inhibitor-1 (PAI1), matrix metalloproteinases-1 (MMP-1), matrix metalloproteinases-3 (MMP-3) and tissue inhibitor of metalloproteinase-2 (TIMP-2). The differently expressed IL18, TGF- $\beta 1$, IL1 $\beta$, TIMP-2, MMP-3, IL8 and MCP1 in the RPE tissues of the aging mice were detected $(P<0.5)$. Most significantly regulated SASP were reserved by three months GSPE supplementation and the visualized data as well as the detailed $P$ values were presented in Fig. 2B. 
To detect the effect of GSPE on the normal and aging ARPE-19 cells, the GSPE in different concentrations $(0.1,1,10,50$ and $100 \mu \mathrm{g} / \mathrm{ml})$ were used. As showed in Fig. 3A and 3B, GSPE failed to affect the cell viability of the normal ARPE-19 cells, however, 10 and more than $10 \mu \mathrm{g} / \mathrm{ml}$ of GSPE significantly improved the cell viability of the aging RPE cells. When the NAD + contents and NAMPT expressions in the aging ARPE-19 cells were considered, a significant deceased NAD + and NAMPT were observed in the aging RPE cells and these two NAD + metabolism markers were up-regulated with $10 \mu \mathrm{g} / \mathrm{ml}$ or higher concentrations of GSPE treatments $(P<0.001$, Fig. 3C and 3D). In advanced experiment about the $\beta$-gal activity, it was found that $\beta$-gal activity was significantly increased in the aging RPE cells and the activity was alleviated by $10 \mu \mathrm{g} / \mathrm{ml}$ or higher concentrations of GSPE treatment $(P<0.001$, Fig. 3E). The effects of GSPE on the cell viability, NAD + metabolism and $\beta$-gal activity were consistent and the concentration of $10 \mu \mathrm{g} / \mathrm{ml}$ would be used in the following experiments.

GSPE alleviated mitochondrial homeostasis, barrier function and SASP of aging RPE cells

As NAD + metabolism was closely related with the mitochondrial homeostasis, the mtDNA lesions were detected with a classical PCR based method and the mitochondrial membrane permeability was analyzed. As showed in Fig. 4A, GSPE treatment significantly alleviated the mtDNA lesions, which was significantly increased in the aging RPE cells $(P<0.001)$. Increased mitochondrial membrane permeability was related with the worsen mitochondrial function and it would demonstrate more monomers $\mathrm{J} 1$ staining in the cells. After treatment with GSPE, the damaged mitochondrial membrane permeability was significantly improved $(P<0.001$, Fig. 4B). Considering that the physiological functions of RPE cells included barrier function and growth factors secretions, the effects of GSPE treatment on the barrier function and SASP on aging RPE cells were reported in this study. Both the barrier related hallmark (ZO1) and barrier function (TEER) were detected in this study and it was reported that GSPE could significantly improve the expression of ZO1 and the reinstate the TEER in the aging RPE cells $(P<0.05$, Fig. $4 C$ and $4 D)$. The abnormal growth factors secretions of RPE cells were related with the incidence of retinal disease and SASP related factors were reported to be significantly expressed in the RPE cells of the aging mice. In this study, significantly expressed TGF- $\beta 1$, IL1 $\beta$, IL6, IL8, MCP-1, G-CSF, CXCL2, MMP3 and TIMP2 was detected $(P<0.05)$. Comparing with the aging RPE cells, GSPE could significantly alleviate the expressions of most dysregulated SASP factors $(P<0.05)$.

GSPE regulated NAMPT/SIRT1 pathway in aging RPE cells

As reported in the in Fig. 5A, the protective effects of GSPE on cellular senescence in aging RPE cells was blocked by NAMPT inhibitor, Fk866, while the supplementation of NMN significant reinstate the protective effect. Advanced study on the NAD + status demonstrated that GSPE demonstrated potential anti-aging effect through up-regulation of NAD + content (Fig. 5B). As NAD + was an important promotor of SIRT1, the effects of GSPE on the NAMPT/NAD+/SIRT1 pathway in aging RPE cells were detected. It was found that GSPE treatment significantly up-regulated the expression of SIRT1 and this regulation effects were modified by NAMPT inhibitor, Fk866, and NAD + precursor, NMN (Fig. 5C). 
GSPE alleviated the inflammasome activation through SIRT1 in aging RPE cells

Inflammasome was associated with the incidence of various of degenderize retinal diseases, the effects of GSPE on the inflammasome activation and potential molecular mechanism was studied in this study. As showed in Fig. 6A, significantly increased NLRP3 was detected in the aging RPE cells and GSPE treatment reduced the NLRP3 expression after 24 or more hours treatment $(P<0.001)$. In the following experiments, 24 hours of GSPE treatment was used in the advanced mechanism study. As the regulation effect of SIRT1 was reported in the previous experiments, the potential molecular mechanism of GSPE up-regulating SIRT1 and inhibiting inflammasome activity was studied. The treatment of GSPE significant extenuate inflammasome activity and the key compounds, including NLRP3, ASC and caspase-1, were significant alleviated by GSPE treatment in the aging RPE cells. While the regulated NLRP3 pattern was suppressed by SIRT1 inhibitor, EX-527 treatment and the detailed data was presented in Fig. 6B. IL-1 $\beta$ and IL-18 were two cytokines related with the NLRP3 activation and it was found that the inhibitory effects of GSPE treatment on the IL1 $\beta$ and IL-18 secretions was disturbed by the SIRT1 inhibitor, EX-527 (Fig. 6C).

\section{Discussion}

Cellular senescence was a major risk factor in the development of degenerative retinal disorders [18]. In this current study, GSPE significantly improve the NAMPT and NAD + content in aging mice and thus alleviated the RPE cellular senescence. In advanced in-vitro studies, GSPE could be an activator of NAMPT and thus relieved $\mathrm{H}_{2} \mathrm{O}_{2}$ induced NAD + depression. Thus, detection the SASP in in-vitro aging model provided us knowledge in the understanding of the anti-aging role of GSPE and following detailed pathological mechanism analyses demonstrated that GSPE demonstrated the protective effects in aging RPE cells through NAMPT/SIRT1/NLRP3 pathway.

AMD was the most common irreversible cause of severe vision loss among the elderly in developed countries. Advanced age was one of the strongest risk factors of the AMD, thus cellular senescence might be associated with the development of AMD [19]. The detailed mechanism of the cellular senescence on AMD remained unclear. The decline of NAMPT and its metabolic product, NAD+, was reported to be related with the aging progresses in different organs and cells, including RPE [19]. Exogenous NAD + precursor, NMN, demonstrated a significant protective role in RPE cellular senescence [20]. NAMPT, which was the rate-limiting enzyme of NAD + synthesis and a potential target in the treatment of cellular senescence, might help to improve NAD + concentration and thus played an important role in the field of anti-aging. GSPE, which was an extract of grape seed, was rich in polyphenolic bioflavonoid. In this current study, it was found that GSPE could significantly improve the NAMPT and NAD + content and thus protected RPE from cellular senescence. NAD + improvement had been regarded as an important approach, however, most commonly used NAD + supplement, NMN, was relatively expensive. Our study provided an economic NAD + improvement therapy through up-regulation of the NAMPT expression in RPE tissues. The results in this study highlighted the importance to screen more anti-aging natural 
extracts and would help improve our understanding in their effects in aging related degenerative disorders.

NAD + improvement could effetely improve various degenerative disorders, however, previous studies have mainly focused on the regulation of NAD + metabolic disorders in retinal nerve cells, while less attention has been paid to RPE cells. Considering that RPE cellular senescence was a key process of various degenerative diseases [21], any treatment targeting RPE cellular senescence would provide huge effect in the management of these disorders. Rather than simply detecting the up-regulation of NAMPT and NAD + by GSPE treatment, more detailed molecular mechanism was studied. SIRT1 functioned based on the NAD + activity and provided remarkable protections for retinal degenerative disorders [22]. Based on the in-vitro experiments and molecular biological data, it was found that GSPE application could significantly up-regulate the SIRT1 level [23]. Inhibition of SIRT1 depleted the protective effect of GSPE significantly and it provided us the confirmation that SIRT1 was an important connection between GSPE treatment and the anti-aging effects in the RPE cells. As NAD + was a powerful activator of SIRT1, it was easy for us to understand the extract molecular mechanism for the protective effects of RPE cellular senescence: the GSPE treatment significantly up-regulated the NAMPT level in the aging RPE cells and thus improve the production of NAD+. Up-regulated NAD + improved the expression of SIRT1, which was a key factor in the anti-aging function during NAD + intervention. This study presented a simple but clear clue of the anti-aging effect of GSPE in RPE cellular senescence, however, the complexed NAD + and SIRT1 regulation system needed to be studied in more advanced experiments. For instance, NAMPT could transform Nicotinamide (NAM) into NMN and NAMPT up-regulation might lead to decreased NAM. NAM was an inhibitor of SIRT1 [24] and NAM down-regulation might be associated with SIRT1 activator. More detailed experiments about the contributions of NAD + metabolism and SIRT1 activity on the RPE cellular senescence were to be conducted.

Cellular senescence would also lead to abnormal expression of inflammatory cytokines and this phenomenon was defined as SASP [25]. SASP was composed of a series of cytokines such as proinflammatory cytokines, growth factors, chemokines and matrix remodeling enzymes and these factors could cause chronic low-grade inflammation and accelerate the senescent progresses in both cells and organs [26]. More and more evidences show that the chronic low-grade inflammation was one of the most important manifestations of AMD [27]. Although the precise mechanism has not been determined so far, we can speculate that SASP was one of the important links between aging and AMD. In this current study, the SASP markers were significantly increased in the aging mice and senescent RPE cellular models and it provided the evidences that SASP could be regarded as a key biological process for AMD development. Advanced experiments demonstrated that GSPE could reduce SASP status significantly. As SASP was both a hallmark and a promoter of cellular senescence, GSPE could efficiently alleviate the retinal degenerative disorders through moderating the RPE cellular senescence related SASP.

The physiological functions of RPE cells included growth factors secretion, the blood-retinal barrier (BRB) maintenance and outer disc membrane phagocytosis $[28,29]$. Pathological stress, such as aging, would lead to the loss of physiological functions and thus cause advanced pathological changes. Abnormal 
growth factors secretion of RPE was one of the most common pathological progresses and it would leaded to neovascularization in choroidal (wet AMD) and retina (proliferative DR)[30]. RPE was the outer BRB and impaired RPE tight junction would cause retinal edema, such as macular edema secondary to DR [31]. Both neovascularization and retinal edema were two important causes of blindness for patients with retinal disorders. In this current study, it was found that GSPE treatment could moderate the abnormal SASP as well as impaired BRB through alleviating the RPE cellular senescence. Thus, GSPE supplement could be a potential treatment for the AMD and DR cases. More advanced experimental studies on diseases animal models and clinical trials on patients with the retinal disease would help us to understand the drug development potential of GSPE.

Based on the experimental data, two important cytokines, IL1 $\beta$ and IL8, were alleviated by GSPE treatment in both in-vivo and in-vitro aging models. Interestingly, these two cytokines were key effectors in the inflammasome activation [32,33]. Inflammasome was a complex composed of multiple proteins, which could activate the protease caspase-1. Caspase-1 protease cleaved pro-IL-1 $\beta$ and pro-IL-18, activated them, and released active IL-1 $\beta$ and IL-18. As reported in previous studies, inflammasome was activated in degenerative retina, including AMD, DR and glaucoma [34, 35]. Previous studies about the effects of SIRT1 on the inflammation activation demonstrated that SIRT1 could moderate the inflammation activity significantly. Based on the results in a myocardial ischemia and reperfusion $(I / R)$ mice model, SIRT1 agonist could reduce NLRP3 inflammasome activation during I/R [36]. Combination the existing knowledge and experimental data in this study together, we could conclude that SIRT1 induced by GSPE was associated with the moderation of inflammasome activation during RPE cellular senescence. Considering that mounting evidences were reported on the relationship between inflammasome and cellular senescence in degenerative diseases, the findings in this study provided a new viewpoint on the crosstalk on the NAD + metabolism and SIRT/NLRP3 pathway in the RPE cellular senescence.

In conclusion, this study has uncovered the protective effects of GSPE on the RPE cellular senescence through up-regulation of NAMPT and improvement of NAD + concentration. NAD + induced SIRT1 expression and then inhibiting the activation of inflammasome complex during RPE aging development. GPSE supplement provides a new potential therapeutic method in RPE cellular senescence associated retinal degenerative disorders.

\section{Declarations}

Ethics approval and consent to participate: The animal protocol was approved by the Animal Ethics Committee of Zhengzhou University.

Consent for publication: Not applicable.

Availability of data and materials: The datasets during and/or analysed during the current study available from the corresponding author on reasonable request. 
Competing interests: Yan Wu is the founder of the Mosi Biotech Company. The other authors report no conflicts of interest.

Funding: This work was supported in whole or in part by National Nature Science Foundation Project for Young Scientists of China (Grant No. 81700804), the Foundation for Young Medical Talents of Jiangsu Province (Grant No. QNRC2016211), Scientific Research Project of Jiangsu Health Commission (Z2019044) and Youth Project of Henan Provincial Health and Health Commission, Ministry of Education (SB201902008).

Authors' contributions: Yan Wu, Sanyou Dai and Yang Long contributed equally to this work; Yan Wu, Guangming Wan, Wencui Wan and Wei Zhu designed the research study; Yan Wu, Sanyou Dai, Long Yang, Hongzhuo Liu, Weiwei Wan, Wencui Wan and Wei Zhu performed the research; Sanyou Dai, Long Yang, Hongzhuo Liu and Weiwei Wan contributed new reagents and analytic tools; Yan Wu, Sanyou Dai, Long Yang, Hongzhuo Liu, Weiwei Wan, Guangming Wan, Wencui Wan and Wei Zhu analyzed the data and wrote the manuscript; All authors have read and approve the final manuscript.

Acknowledgements: Not applicable.

\section{References}

1. Sreekumar PG, Kannan R. Mechanisms of protection of retinal pigment epithelial cells from oxidant injury by humanin and other mitochondrial-derived peptides: Implications for age-related macular degeneration. Redox Biol 2020:101663.doi:10.1016/j.redox.2020.101663.

2. Corbelli E, Parravano M, Sacconi R, Sarraf D, Yu SY, Kim K, Capuano V, Miere A, Souied E, Varano M, et al. Prevalence and Phenotypes of Age-Related Macular Degeneration in Eyes With High Myopia. Invest Ophthalmol Vis Sci. 2019;60(5):1394-402. doi:10.1167/iovs.18-25534.

3. Hyttinen JMT, Viiri J, Kaarniranta K, Blasiak J. Mitochondrial quality control in AMD: does mitophagy play a pivotal role? Cell Mol Life Sci. 2018;75(16):2991-3008. doi:10.1007/s00018-018-2843-7.

4. Ueda K, Kim HJ, Zhao J, Song Y, Dunaief JL, Sparrow JR. Iron promotes oxidative cell death caused by bisretinoids of retina. Proc Natl Acad Sci U S A. 2018;115(19):4963-8.

doi:10.1073/pnas.1722601115.

5. Wolk A, Upadhyay M, Ali M, Suh J, Stoehr H, Bonilha VL, Anand-Apte B. The retinal pigment epithelium in Sorsby Fundus Dystrophy shows increased sensitivity to oxidative stress-induced degeneration. Redox Biol 2020:101681.doi:10.1016/j.redox.2020.101681.

6. Jadeja RN, Thounaojam MC, Bartoli M, Martin PM. Implications of NAD(+) Metabolism in the Aging Retina and Retinal Degeneration. Oxid Med Cell Longev. 2020;2020:2692794. doi:10.1155/2020/2692794.

7. Wang Y, Yang JQ, Hong TT, Sun YH, Huang HL, Chen F, Chen XJ, Chen HY, Dong SS, Cui LL, et al: RTN4B-mediated suppression of Sirtuin 2 activity ameliorates beta-amyloid pathology and cognitive impairment in Alzheimer's disease mouse model. Aging Cell 2020:e13194.doi:10.1111/acel.13194. 
8. Wang Y, Grenell A, Zhong F, Yam M, Hauer A, Gregor E, Zhu S, Lohner D, Zhu J, Du J. Metabolic signature of the aging eye in mice. Neurobiol Aging. 2018;71:223-33.

doi:10.1016/j.neurobiolaging.2018.07.024.

9. Liebmann JM, Cioffı GA. Nicking Glaucoma with Nicotinamide? N Engl J Med. 2017;376(21):207981. doi:10.1056/NEJMcibr1702486.

10. Mekala NK, Kurdys J, Depuydt MM, Vazquez EJ, Rosca MG. Apoptosis inducing factor deficiency causes retinal photoreceptor degeneration. The protective role of the redox compound methylene blue. Redox Biol. 2019;20:107-17. doi:10.1016/j.redox.2018.09.023.

11. Jadeja RN, Powell FL, Jones MA, Fuller J, Joseph E, Thounaojam MC, Bartoli M, Martin PM. Loss of NAMPT in aging retinal pigment epithelium reduces $N A D(+)$ availability and promotes cellular senescence. Aging. 2018;10(6):1306-23. doi:10.18632/aging.101469.

12. Wang L, Huang W, Zhan J. Grape Seed Proanthocyanidins Induce Autophagy and Modulate Survivin in HepG2 Cells and Inhibit Xenograft Tumor Growth in Vivo. Nutrients 2019, 11(12).doi:10.3390/nu11122983.

13. Ahmad SR, Gokulakrishnan P, Giriprasad R, Yatoo MA. Fruit-based Natural Antioxidants in Meat and Meat Products: A Review. Crit Rev Food Sci Nutr. 2015;55(11):1503-13. doi:10.1080/10408398.2012.701674.

14. Nie Y, Sturzenbaum SR. Proanthocyanidins of Natural Origin: Molecular Mechanisms and Implications for Lipid Disorder and Aging-Associated Diseases. Adv Nutr. 2019;10(3):464-78. doi:10.1093/advances/nmy118.

15. Barbe A, Mellouk N, Rame C, Grandhaye J, Anger K, Chahnamian M, Ganier P, Brionne A, Riva A, Froment $P$, et al. A grape seed extract maternal dietary supplementation improves egg quality and reduces ovarian steroidogenesis without affecting fertility parameters in reproductive hens. PLoS One. 2020;15(5):e0233169. doi:10.1371/journal.pone.0233169.

16. Zhu W, Wu Y, Meng YF, Wang JY, Xu M, Tao JJ, Lu J. Effect of curcumin on aging retinal pigment epithelial cells. Drug Des Devel Ther. 2015;9:5337-44. doi:10.2147/DDDT.S84979.

17. Lin H, Xu H, Liang FQ, Liang H, Gupta P, Havey AN, Boulton ME, Godley BF. Mitochondrial DNA damage and repair in RPE associated with aging and age-related macular degeneration. Invest Ophthalmol Vis Sci. 2011;52(6):3521-9. doi:10.1167/iovs.10-6163.

18. O'Mealey GB, Berry WL, Plafker SM. Sulforaphane is a Nrf2-independent inhibitor of mitochondrial fission. Redox Biol. 2017;11:103-10. doi:10.1016/j.redox.2016.11.007.

19. Marie M, Forster V, Fouquet S, Berto P, Barrau C, Ehrismann C, Sahel JA, Tessier G, Picaud S. Phototoxic damage to cone photoreceptors can be independent of the visual pigment: the porphyrin hypothesis. Cell Death Dis. 2020;11(8):711. doi:10.1038/s41419-020-02918-8.

20. Braidy N, Liu Y. NAD + therapy in age-related degenerative disorders: A benefit/risk analysis. Exp Gerontol. 2020;132:110831. doi:10.1016/j.exger.2020.110831.

21. Wang S, Wang X, Cheng Y, Ouyang W, Sang X, Liu J, Su Y, Liu Y, Li C, Yang L, et al. Autophagy Dysfunction, Cellular Senescence, and Abnormal Immune-Inflammatory Responses in AMD: From 
Mechanisms to Therapeutic Potential. Oxid Med Cell Longev. 2019;2019:3632169. doi:10.1155/2019/3632169.

22. Zhou Y, Wang S, Wan T, Huang Y, Pang N, Jiang X, Gu Y, Luo J, Yang L. Cyanidin-3-O-beta-glucoside inactivates NLRP3 inflammasome and alleviates alcoholic steatohepatitis via SirT1/NF-kappaB signaling pathway. Free Radic Biol Med. 2020. doi:10.1016/j.freeradbiomed.2020.08.006.

23. Cai X, Bao L, Ren J, Li Y, Zhang Z. Grape seed procyanidin B2 protects podocytes from high glucoseinduced mitochondrial dysfunction and apoptosis via the AMPK-SIRT1-PGC-1alpha axis in vitro. Food Funct. 2016;7(2):805-15. doi:10.1039/c5fo01062d.

24. Pan S, Leng J, Deng X, Ruan H, Zhou L, Jamal M, Xiao R, Xiong J, Yin Q, Wu Y, et al. Nicotinamide increases the sensitivity of chronic myeloid leukemia cells to doxorubicin via the inhibition of SIRT1. J Cell Biochem. 2020;121(1):574-86. doi:10.1002/jcb.29303.

25. Dominic A, Banerjee P, Hamilton DJ, Le NT, Abe JI. Time-dependent replicative senescence vs. disturbed flow-induced pre-mature aging in atherosclerosis. Redox Biol 2020:101614.doi: 10.1016/j.redox.2020.101614.

26. Borghesan M, Hoogaars WMH, Varela-Eirin M, Talma N, Demaria M. A Senescence-Centric View of Aging: Implications for Longevity and Disease. Trends Cell Biol. 2020.

doi:10.1016/j.tcb.2020.07.002.

27. Faber C, Juel HB, Jensen BAH, Christensen JP, Prause JU, Thomsen AR, Nissen MH: Chemokine Expression in Murine RPE/Choroid in Response to Systemic Viral Infection and Elevated Levels of Circulating Interferon-gamma. Invest Ophthalmol Vis Sci 2019, 60(1):192-201.doi: 10.1167/iovs.1825721.

28. Kaarniranta K, Pawlowska E, Szczepanska J, Blasiak J. DICER1 in the Pathogenesis of Age-related Macular Degeneration (AMD) - Alu RNA Accumulation versus miRNA Dysregulation. Aging Dis. 2020;11(4):851-62. doi:10.14336/AD.2019.0809.

29. Ghareeb $A E$, Lako $M$, Steel $D H$. Coculture techniques for modeling retinal development and disease, and enabling regenerative medicine. Stem Cells Transl Med. 2020. doi:10.1002/sctm.20-0201.

30. Oubaha M, Miloudi K, Dejda A, Guber V, Mawambo G, Germain MA, Bourdel G, Popovic N, Rezende FA, Kaufman RJ, et al. Senescence-associated secretory phenotype contributes to pathological angiogenesis in retinopathy. Sci Transl Med. 2016;8(362):362ra144.

doi:10.1126/scitranslmed.aaf9440.

31. Kuo C, Green CR, Rupenthal ID, Mugisho 00. Connexin43 hemichannel block protects against retinal pigment epithelial cell barrier breakdown. Acta Diabetol. 2020;57(1):13-22. doi:10.1007/s00592019-01352-3.

32. Rao A, Strauss O, Kokkinou E, Bruchard M, Tripathi KP, Schlums H, Carrasco A, Mazzurana L, Konya V, Villablanca EJ, et al. Cytokines regulate the antigen-presenting characteristics of human circulating and tissue-resident intestinal ILCs. Nat Commun. 2020;11(1):2049. doi:10.1038/s41467-020-15695$x$. 
33. Korhonen E, Bisevac J, Hyttinen JMT, Piippo N, Hytti M, Kaarniranta K, Petrovski G, Kauppinen A. UVB-Induced Inflammasome Activation Can Be Prevented by Cis-Urocanic Acid in Human Corneal Epithelial Cells. Invest Ophthalmol Vis Sci. 2020;61(4):7.doi. 10.1167/iovs.61.4.7.

34. Du J, Wang Y, Tu Y, Guo Y, Sun X, Xu X, Liu X, Wang L, Qin X, Zhu M, et al. A prodrug of epigallocatechin-3-gallate alleviates high glucose-induced pro-angiogenic factor production by inhibiting the ROS/TXNIP/NLRP3 inflammasome axis in retinal Muller cells. Exp Eye Res. 2020;196:108065. doi:10.1016/j.exer.2020.108065.

35. Mat Nor MN, Rupenthal ID, Green CR, Acosta ML. Connexin Hemichannel Block Using Orally Delivered Tonabersat Improves Outcomes in Animal Models of Retinal Disease. Neurotherapeutics. 2020;17(1):371-87. doi:10.1007/s13311-019-00786-5.

36. Han Y, Sun W, Ren D, Zhang J, He Z, Fedorova J, Sun X, Han F, Li J. SIRT1 agonism modulates cardiac NLRP3 inflammasome through pyruvate dehydrogenase during ischemia and reperfusion. Redox Biol. 2020;34:101538. doi:10.1016/j.redox.2020.101538.

\section{Figures}



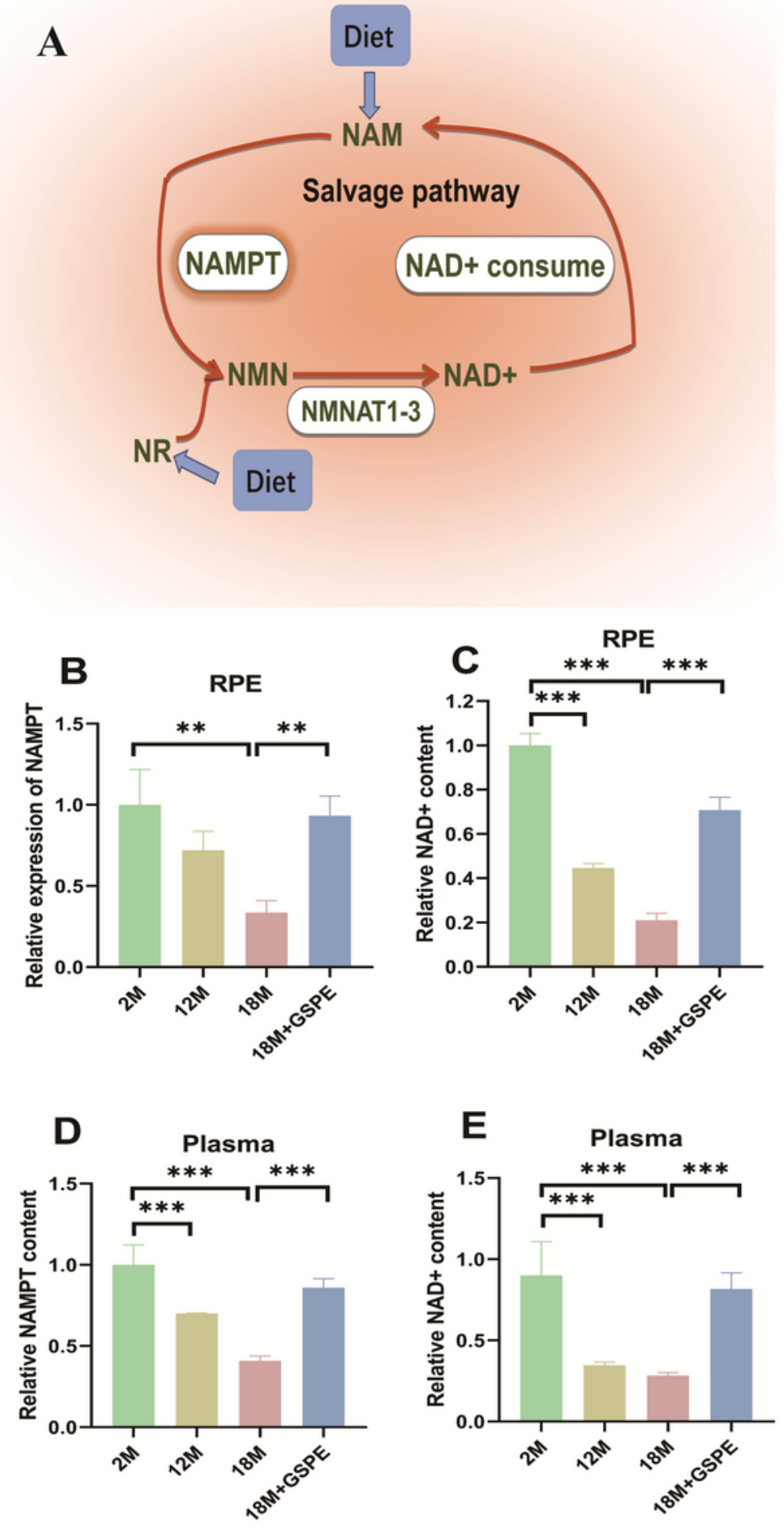

\section{Figure 1}

Decreased NAMPT and NAD+ levels in RPE and plasma could be improved by GSPE treatment in aging mouse. A. The salvage pathway of NAD+ metabolism: NAD+ was transformed into NAM through NAD+ consume, the NAM would become NMN after being catalyzed by NAMPT and NMN would also be transformed into NAD+ by NMNAT1-3 enzyme. B-C: Compared with 2-month aged mice, NAMPT expression is reduced in 18-month aged mice, and NAD+ content is reduced in 12-month and 18-month 
aged mice. After supplementing with GSPE at a concentration of $250 \mathrm{mg} / \mathrm{kg}$ at 15 to 18 months of age, significant increase in NAMPT expression and NAD+ content. $n=3, * *: P<0.01$. D-E: The decrease of plasma NAMPT/NAD + level was related to the increased mouse age. Ingestion of $250 \mathrm{mg} / \mathrm{kg}$ GSPE for three months can significantly improve the content of NAMTP and NAD+ in plasma. $n=3, \star \star$ : $P<0.01$, $\star \star \star$ : $\mathrm{P}<0.001$.

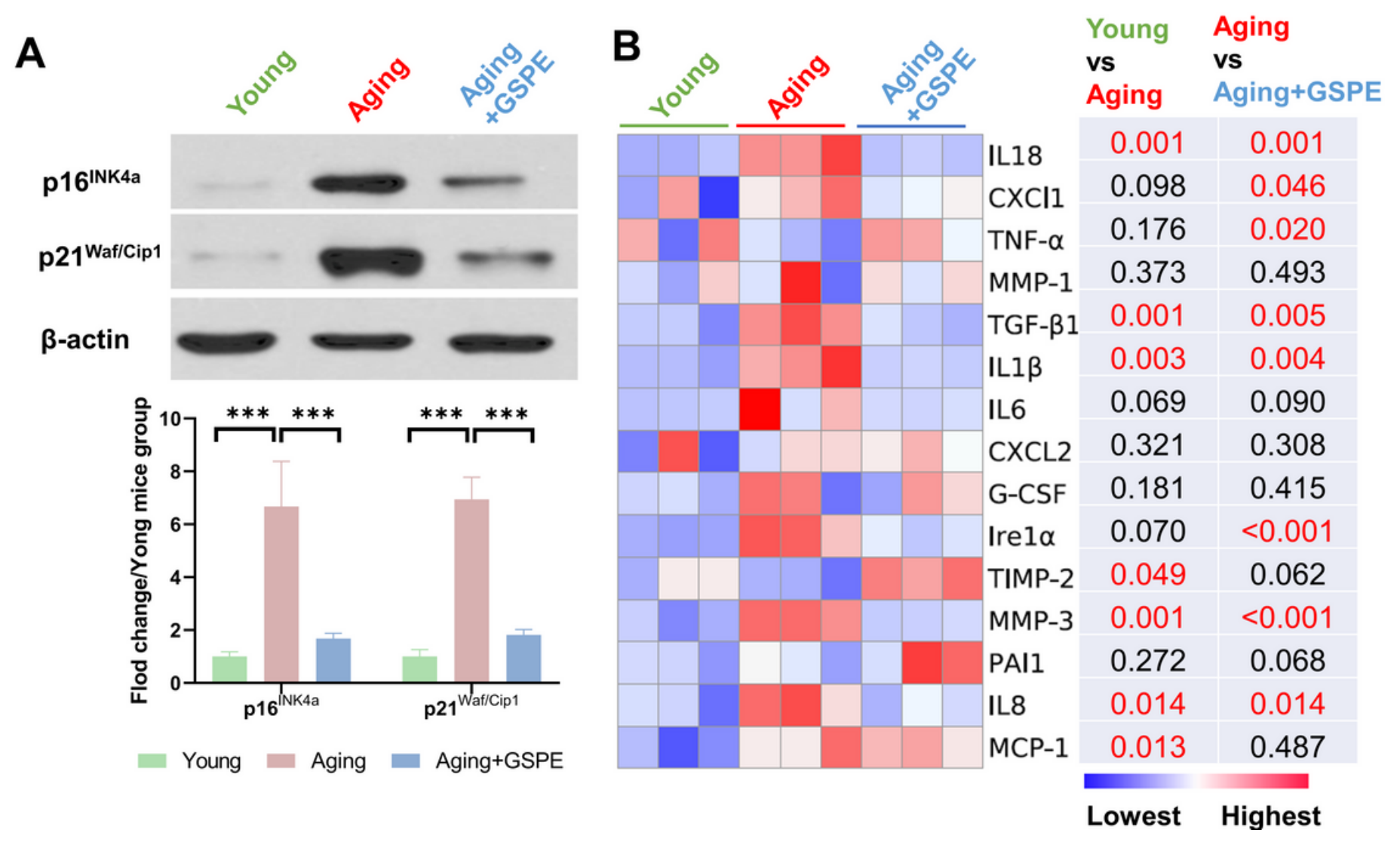

Figure 2

GSPE alleviated aging related cellular senescence and SASP in mouse RPE. A: p16INK4a and p21Waf/Cip1 expressions were increased in the aging mice (18 months aged) and the GSPE in 250 $\mathrm{mg} / \mathrm{kg}$ would decline these cellular senescence biomarkers. The representative western-blot gels and statistical results were presented. $n=3, \star \star *: P<0.001$. B: The SASP factors in the young, aging and aging mice treated with three-month GSPE supplementation. The expressions of TNF- $\alpha$, TGF- $\beta 1$, IL1 $\beta$, IL6, IL8, IL18, MCP-1, G-CSF, CXCl1, CXCL2, Ire1a, PAI1, MMP-1 and MMP-3 were visualized by s heatmap. The lowest expression was marked as blue and the highest expression was marked as red. The P values of comparison of young vs aging as well as aging vs aging mice treated with GSPE were presented and any $P$ values of significant differences were marked red $(P<0.05) . n=3$. 

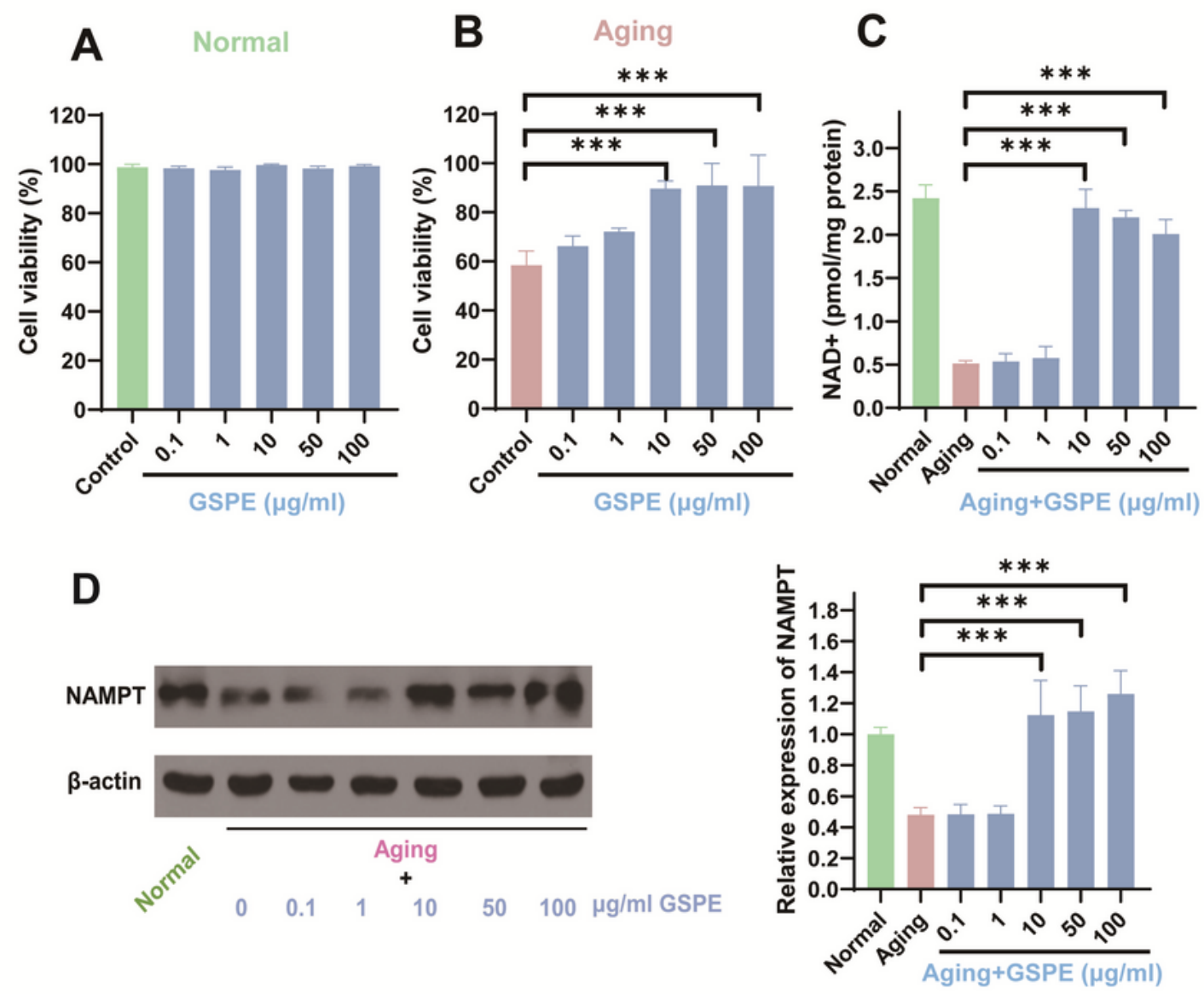

E

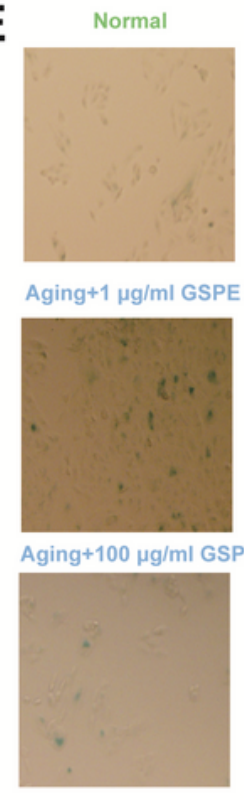

Aging

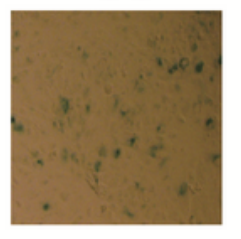

Aging $+10 \mu \mathrm{g} / \mathrm{ml}$ GSPE
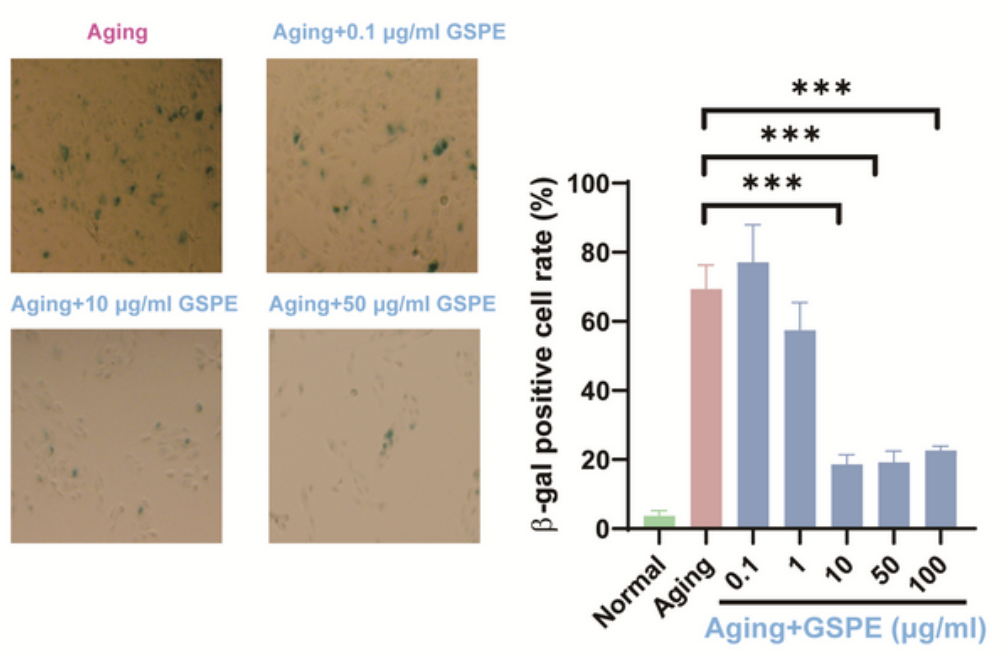

\section{Figure 3}

GSPE extenuated RPE cellular senescence of the in-vitro model. A-B: GSPE failed to affect the cell viability of normal ARPE-19 cells, while GSPE at 10 and $10 \mu \mathrm{g} / \mathrm{ml}$ significantly improved the cell viability of senescent RPE cells. $n=3, * \star *: P<0.001$. C. NAD + content was decreased significantly in senescent RPE cells, and the NAD + content was up-regulated with $10 \mu \mathrm{g} / \mathrm{ml}$ or higher concentrations of GSPE treatments. $n=3, * \star \star: P<0.001$. D: The expression of NAMPT was decreased significantly in senescent RPE 
cells, and it was up-regulated with $10 \mu \mathrm{g} / \mathrm{ml}$ or higher concentrations of GSPE treatments. The representative western-blot gels and statistical results were presented. $n=3$, $\star \star \star: P<0.001$. E: The $\beta$-gal activity is significantly increased in senescent RPE cells, and treatment with GSPE at a concentration of $10 \mu \mathrm{g} / \mathrm{ml}$ or higher reduces the activity. $\mathrm{n}=3, * \star *: \mathrm{P}<0.001$.

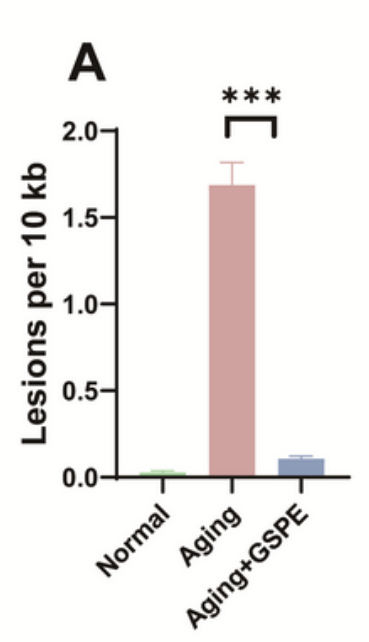

C

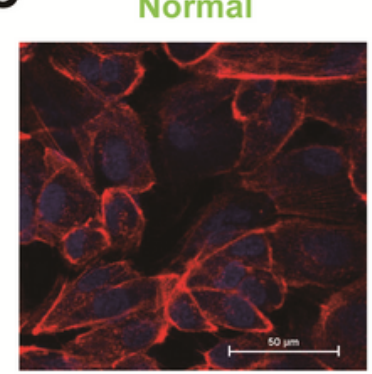

Aging+GPSE

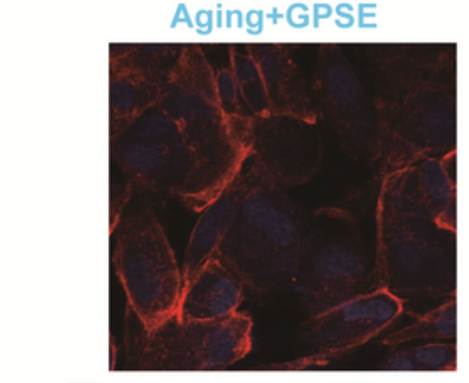

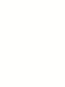

B

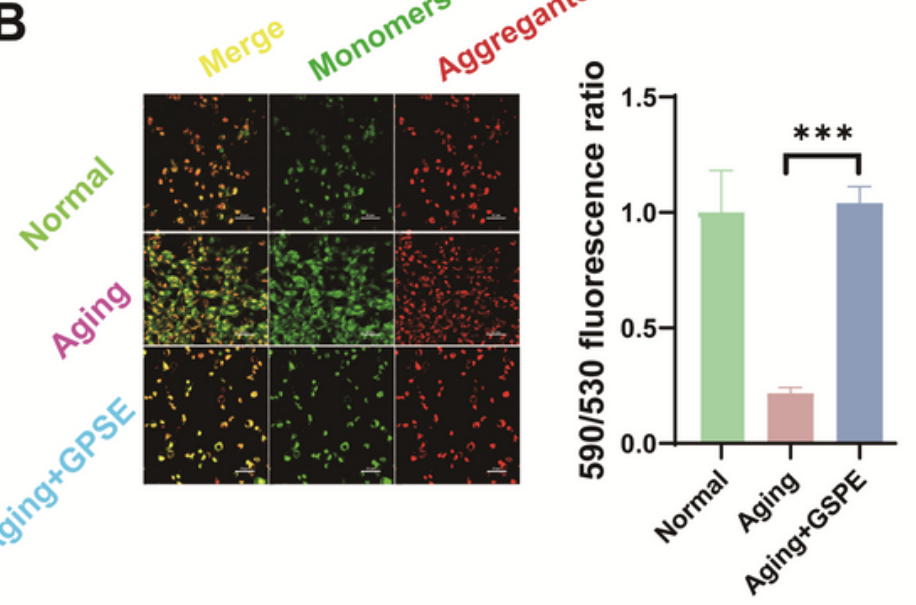

Aging
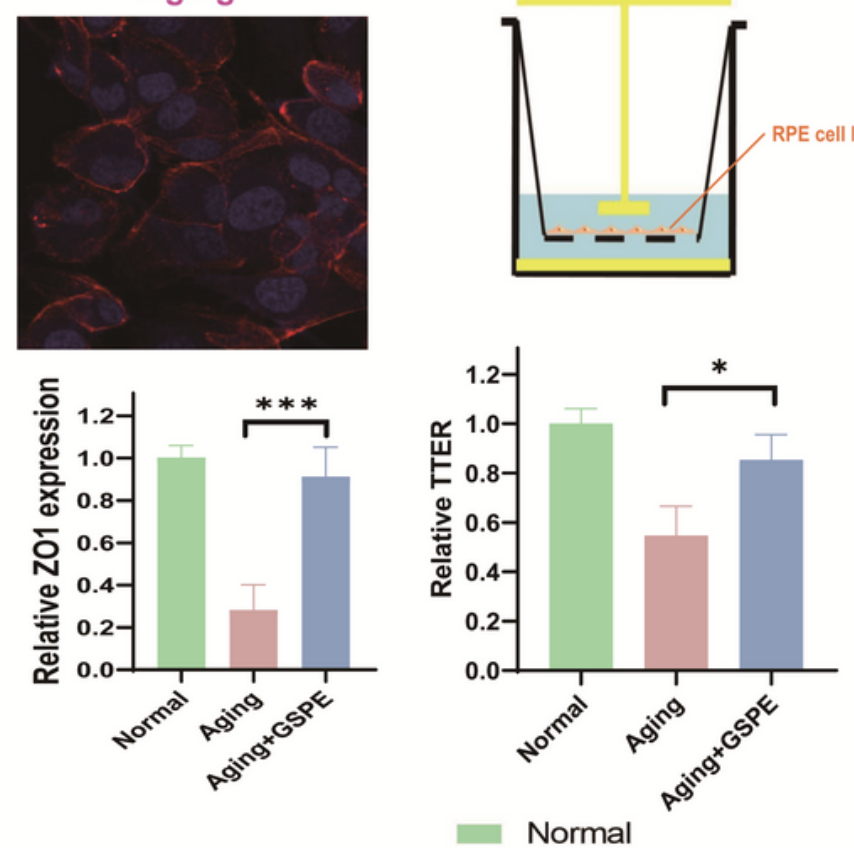

Normal

- Aging

Aging+GSPE
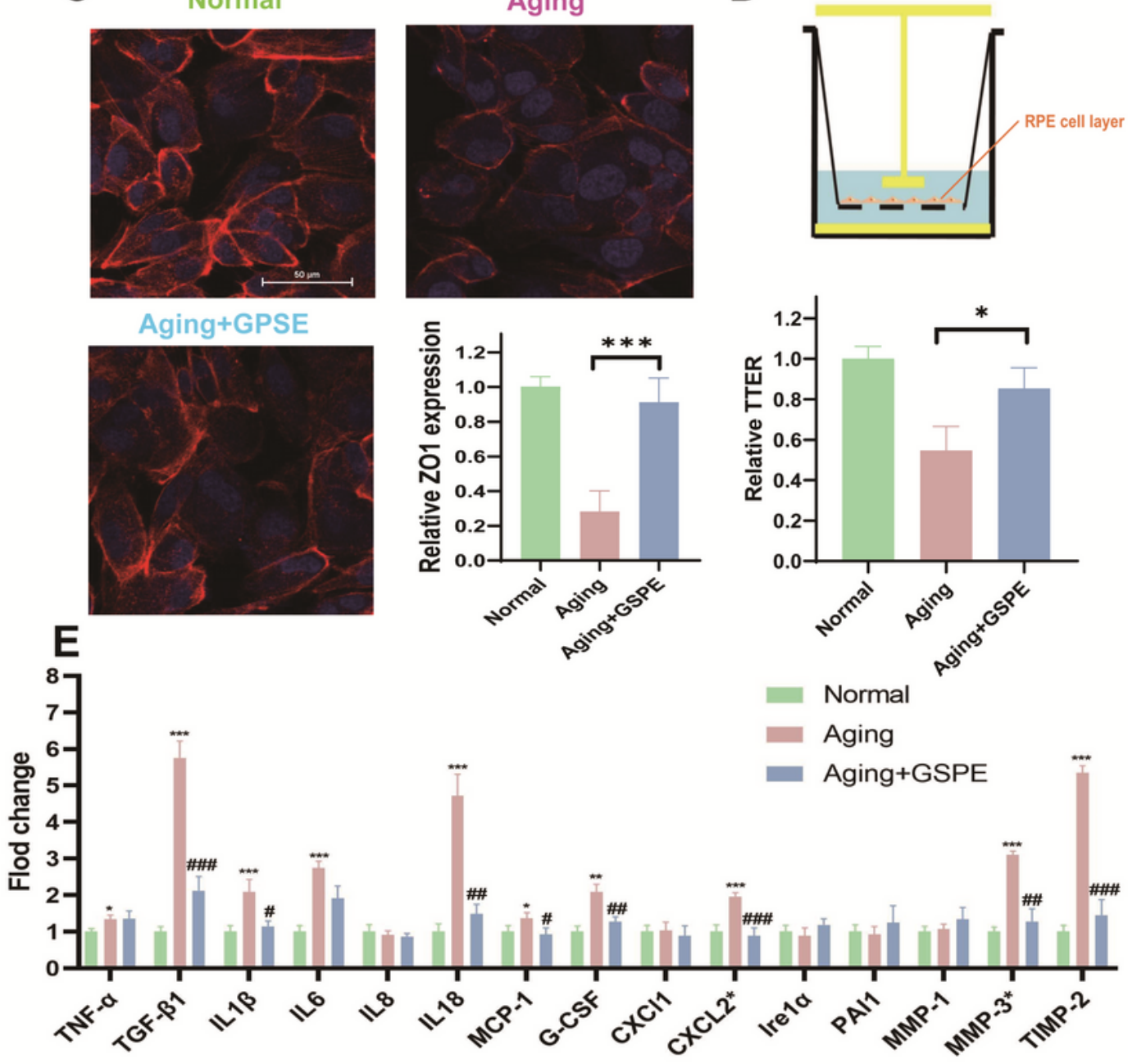

Figure 4 
GSPE alleviated mitochondrial homeostasis, barrier function and SASP of aging RPE cells. A: GSPE treatment significantly alleviated the mtDNA lesions, which was significantly increased in the aging RPE cells. $n=3, * * *$ : $P<0.001$. B: After GSPE treatment, less monomer J1 stained, and the permeability of the damaged mitochondrial membrane was significantly improved. The $\mathrm{J} 1$ monomers were stained green and $\mathrm{J} 1$ aggregates were stained red. $n=3, P<0.001$. C. GSPE could significantly improve the expression of ZO1 in the aging RPE cells. ZO1 was stained read and the nuclear was stained blue with DAPI. $n=3, P$ $<0.001$. D: GSPE treatment significantly improve the reinstate the TEER in the aging RPE cells. $n=3$, *: $P<0.05$. E: Significantly expressed TGF- $\beta 1$, IL1 $\beta$, IL6, IL8, MCP-1, G-CSF, CXCL2, MMP3 and TIMP-2 was detected and GSPE could significantly alleviate the expressions of most dysregulated SASP factors comparing with the aging RPE cells. *: the comparison of aging and normal groups. \#: the comparison of

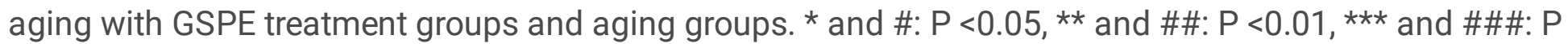
$<0.001$.

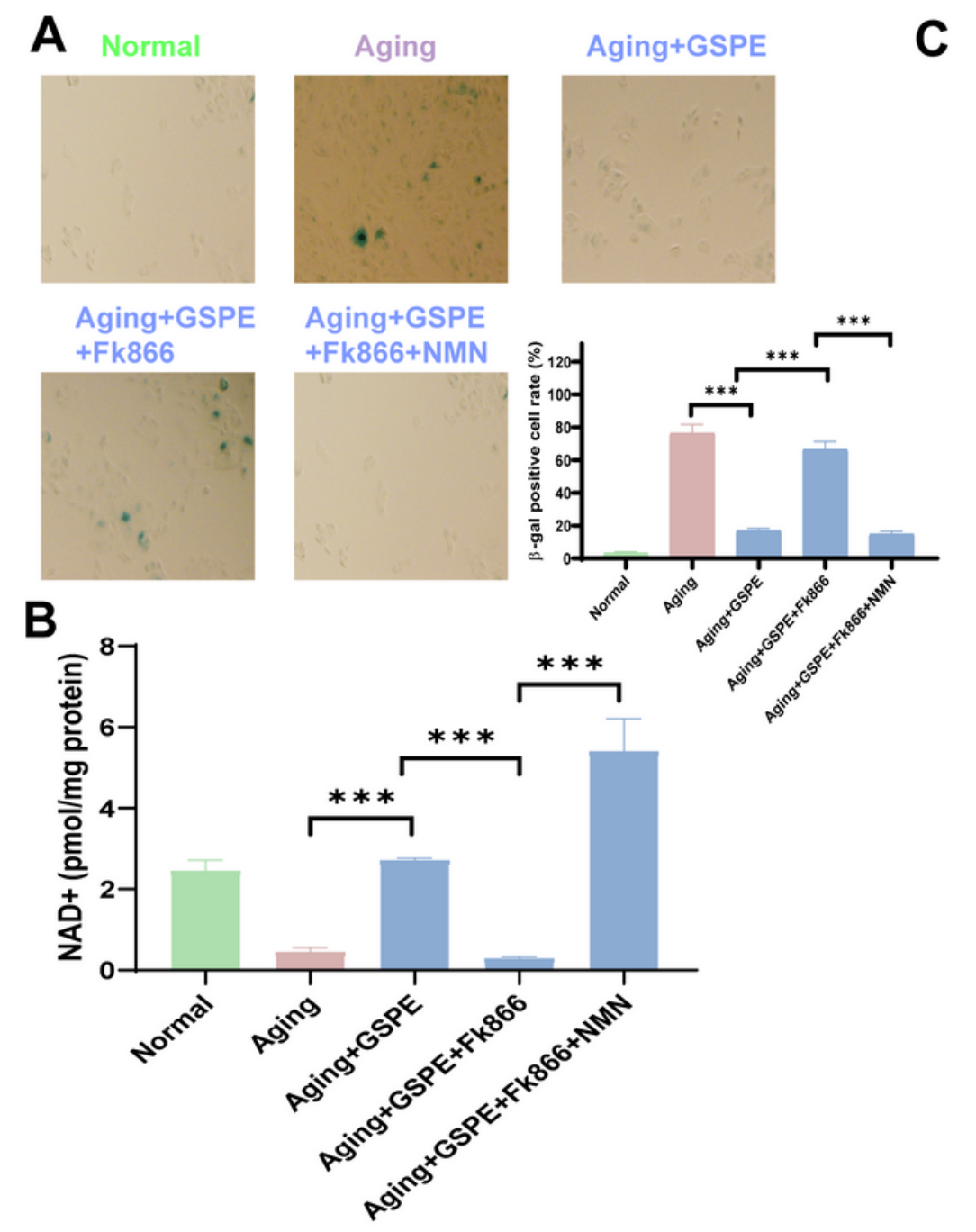

Aging+GSPE +Fk866

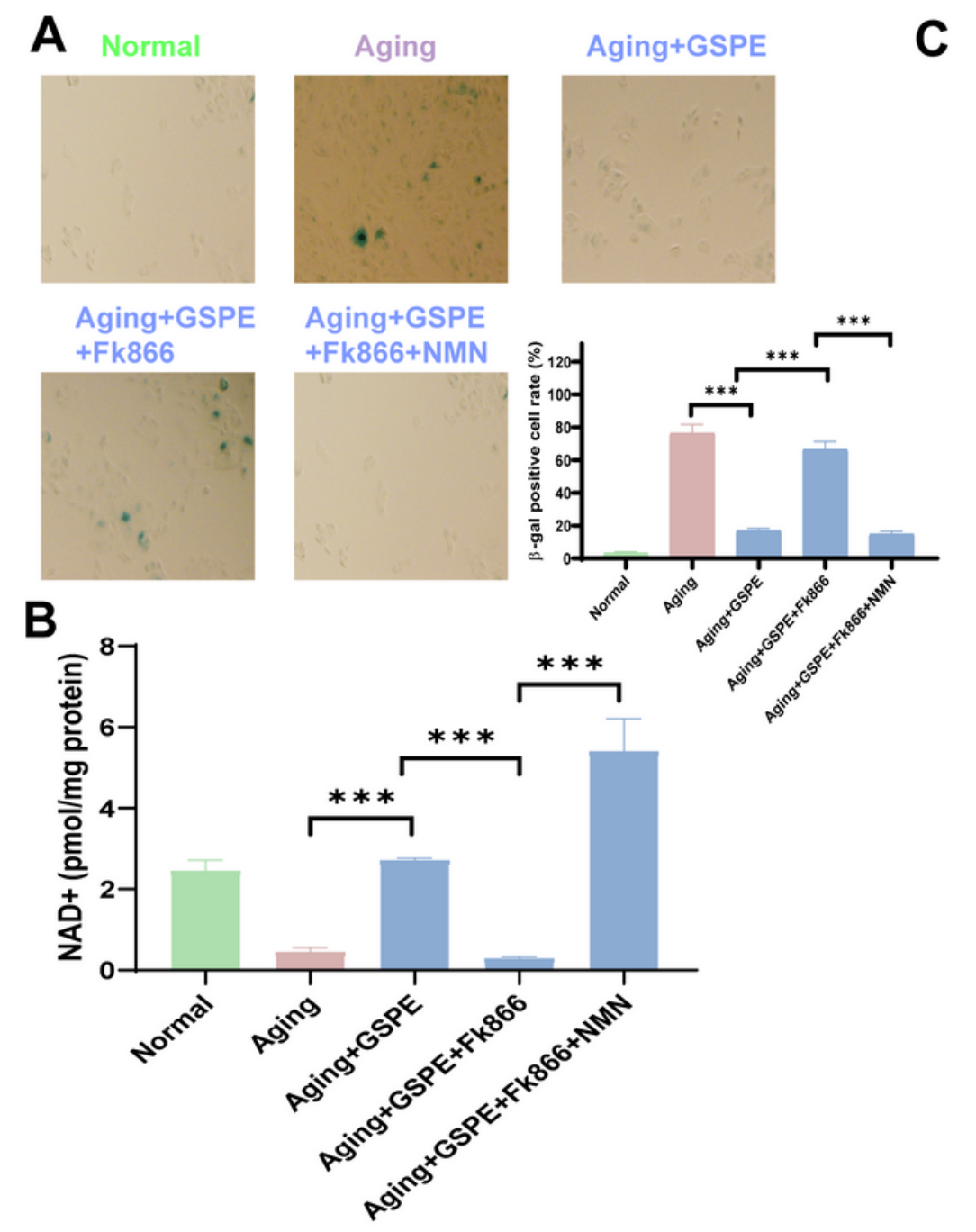

B

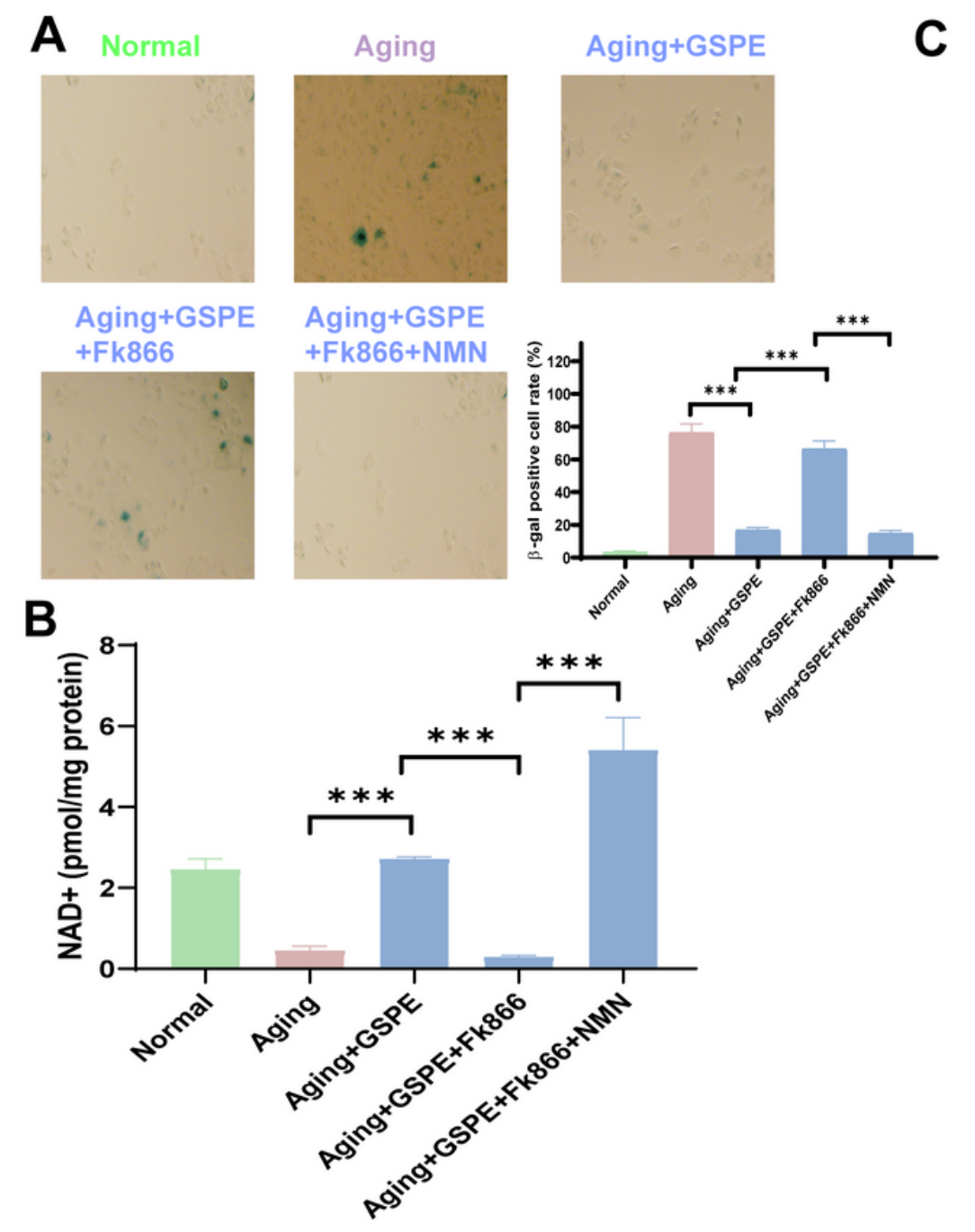

Aging+GSPE
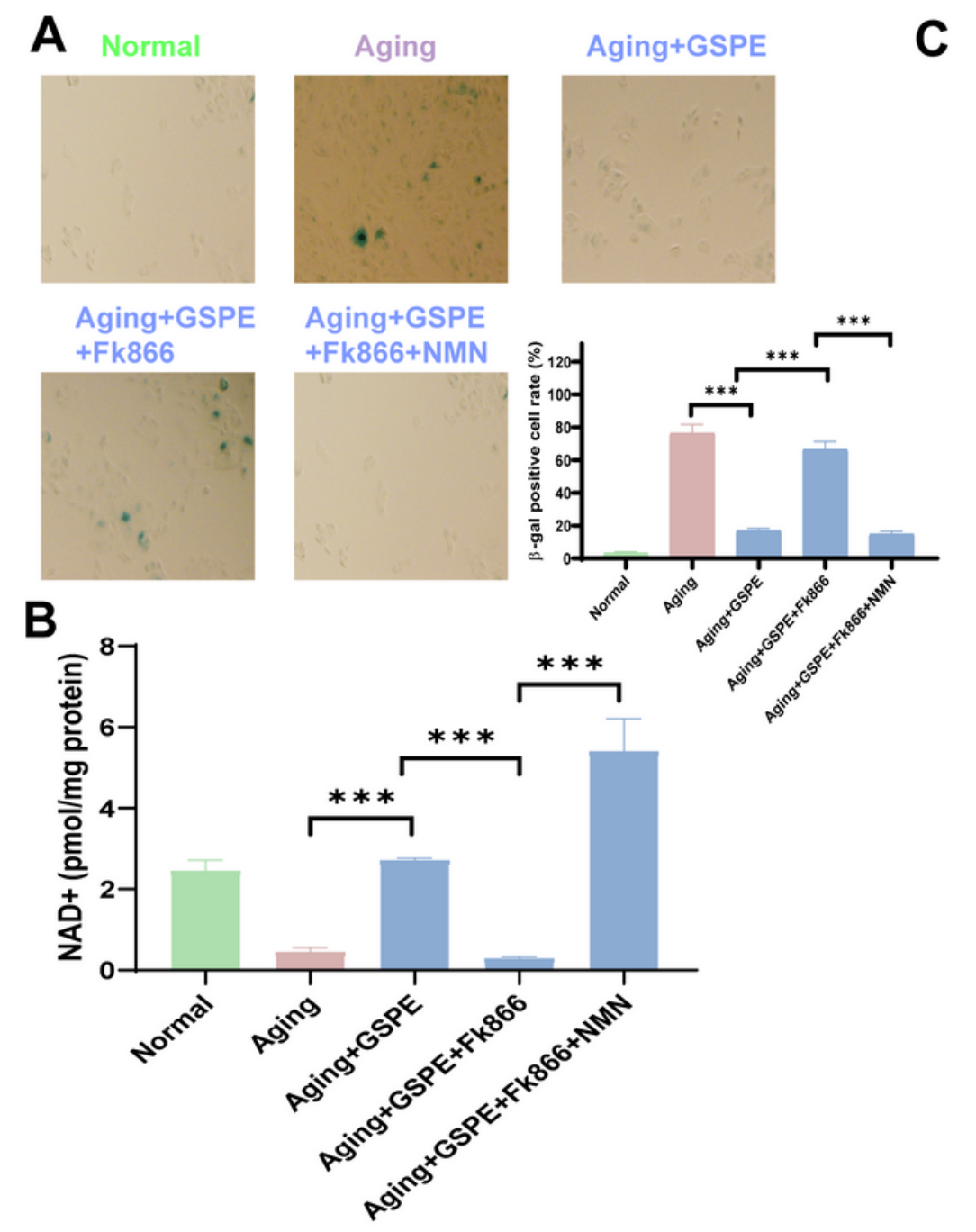

\section{Figure 5}

GSPE regulated NAMPT/SIRT1 pathway in aging RPE cells. A: The NAMPT inhibitor Fk866 blocked the protective effect of GSPE on senescent RPE cells, while supplementing with NMN significantly restored the protective effect. $n=3, P<0.001$. B: GSPE demonstrated potential anti-aging effect through up- 
regulation of $N A D+$ content. $n=3, P<0.001$. C: GSPE treatment significantly up-regulated the expression of SIRT1 and this regulation effects were modified by NAMPT inhibitor, Fk866, and NAD+ precursor, NMN. The representative western-blot gels and statistical results were presented. $n=3, P<0.001$.
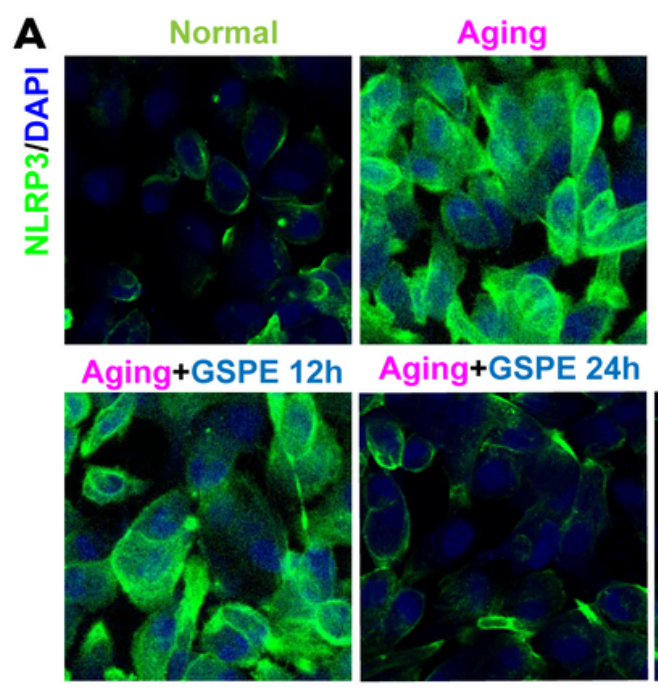

Aging+GSPE 48h
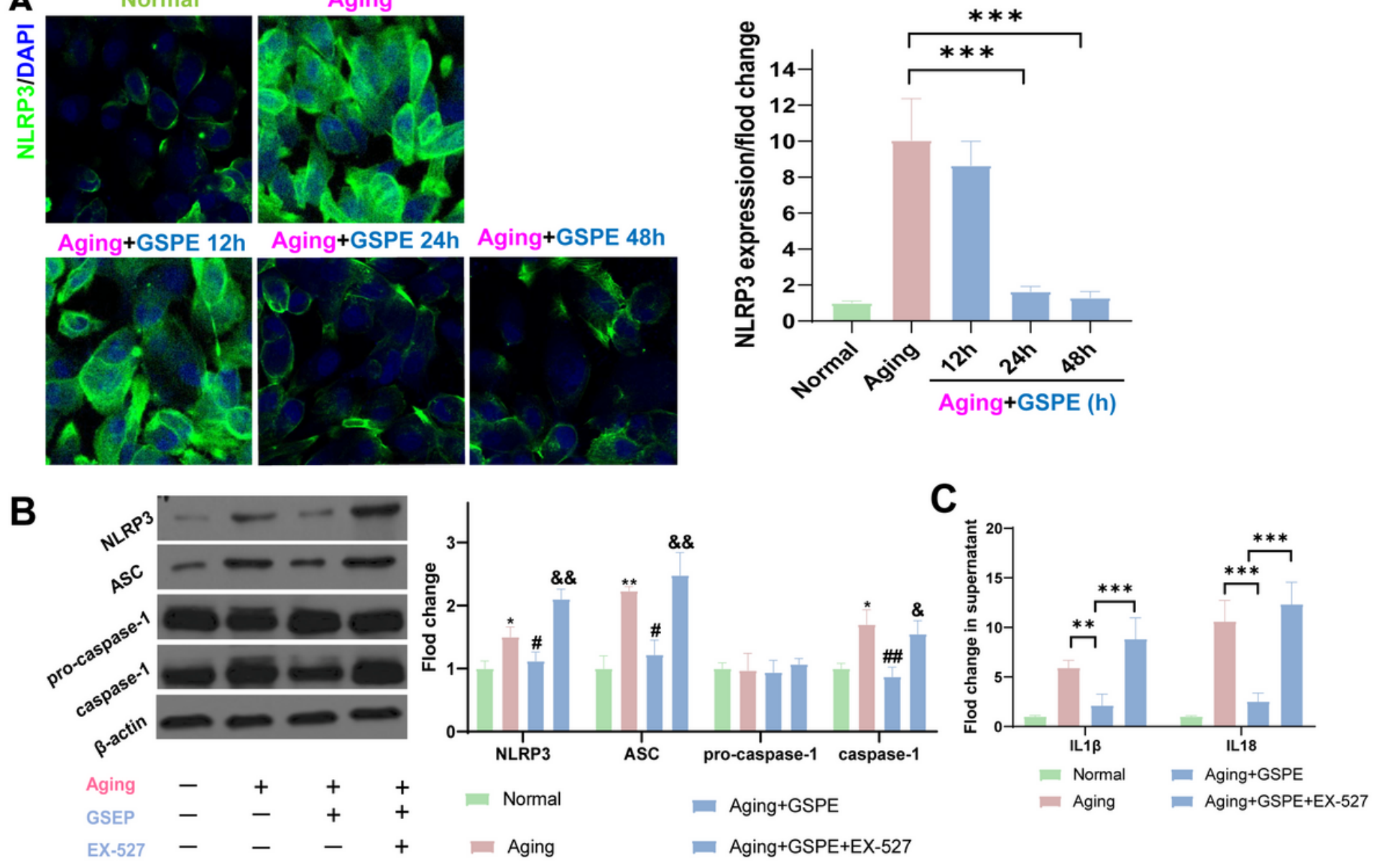

Figure 6

GSPE alleviated the inflammasome activation through SIRT1 in aging RPE cells. A: significantly increased NLRP3 was detected in the aging RPE cells and GSPE treatment reduced the NLRP3 expression after 24 or more hours treatment. The NLRP3 was stained greed and the nuclear was stained blue with DAPI. $n=3$, $P<0.001$. B: The treatment of GSPE significantly reduced the activity of inflammasomes such as NLRP3, ASC and caspase-1, and was significantly relieved in aging RPE cells. While SIRT1 inhibited the NLRP3 regulation mode, it was processed with EX-527 and provided detailed data. The representative westernblot gels and statistical results were presented. *: the comparison of aging and normal groups. \#: the comparison of aging with GSPE treatment groups and aging groups, \&: the comparison of aging with GSPE+EX-527 treatment groups and aging with GSPE groups. $n=3, *$, \# and \&: $P<0.05$, **, \#\# and \&\&: $P$ $<0.01$, $* \star \star, ~ \# \# \#$ and \&\&\&: P $<0.001$. C: the inhibitory effects of GSPE treatment on the IL1 $\beta$ and IL-18 secretions was disturbed by the SIRT1 inhibitor, EX-527. $n=3, P<0.001$.

\section{Supplementary Files}


This is a list of supplementary files associated with this preprint. Click to download.

- SupplementalTable1.docx 